\title{
Development of Analog to Digital Interface for Scalable Ultrasonic Sensor Array
}

\author{
Yuichi Morita, Sota Kono, and Akira Yamawaki* \\ Kyushu Institute of Technology, Kitakyushu, 804-8550 Japan \\ *Corresponding Author: yama@ecs.kyutech.ac.jp
}

\begin{abstract}
We have proposed the organizing method of the 2-D ultrasonic sensor array with the constant number of the wire cables between the sensing part and the data processing part independent to the number of sensors. This paper develops an analog-digital interface between the ultrasonic sensors to the data sending part to realize our proposed organization. To make analog circuits simple, we use only amplifier circuits without smoothing circuits and comparators generally used. Outputs from ultrasonic sensors are amplified to be over $V_{I H}$ of the flip-flops. The flip-flops capture the amplified signals as set pluses. To verify the practical execution, we measure relative distance of each object. The objects are placed in 1 $\times 4$ and $2 \times 2$. We try 16 patterns in each placement. Each sensor is connected to flip-flops in the FPGA through analog circuits. As a result, we confirm that distance can be measured by the simplified A/D structure only with amplifier circuits and flip-flops.
\end{abstract}

Keywords: 2-D ultrasonic sensor array, analog-digital interface, amplifier circuit, flip-flop.

\section{Introduction}

2-D ultrasonic sensor array realizes 3-D imaging system and many studies have been reported to improve the resolution ${ }^{(1,2)}$. One of them tried to combine sensor array with integrated circuits, which allows for better utilization of large array ${ }^{(3)}$. As post-processing, imaging technique using sensor array are improved. One study reported that large amount of data from array can be processed with GPU ${ }^{(4)}$. In this techniques, focused beams to any points can be synthesized and 3D image can be reconstructed with high resolution. However, the number of sensors is limited because the number of cables to the backend that process a large number of dataset increases ${ }^{(5)}$.

To solve this problem, we have proposed a new frontend structure of 2-D ultrasonic sensor array that has constant cables even if many sensors are placed in 2-D. Fig. 1 shows the structure. In this structure, ultrasonic waves transmitted from speakers reflect at the surface of the object and arrive at each sensor. Outputs from each sensor are input into flipflops through amplifiers. These flip-flops construct vertical shift registers and horizontal sift registers. These flip-flops shift out each value in the vertical and horizontal directions. Thus an arrival map in one time can be get. Comparing maps, the time difference of each arrival wave can be acquired and the distance can be calculated by the acquired time difference. Although it take a certain time to get all values in a map, we showed that short range measurement and 3-D image with enough frame rate can be realized by adjusting the frequency of the shift register ${ }^{(6)}$.

As a first step to realize our proposed organization, we develop the A/D interface between the ultrasonic sensors to the data sending part in this paper. First, outputs from ultrasonic sensors are processed in the analog circuits. The flip-flop in the digital circuits capture the signals from analog circuits. Analog circuits often are influenced by external factors such as noises and temperature. Complex analog

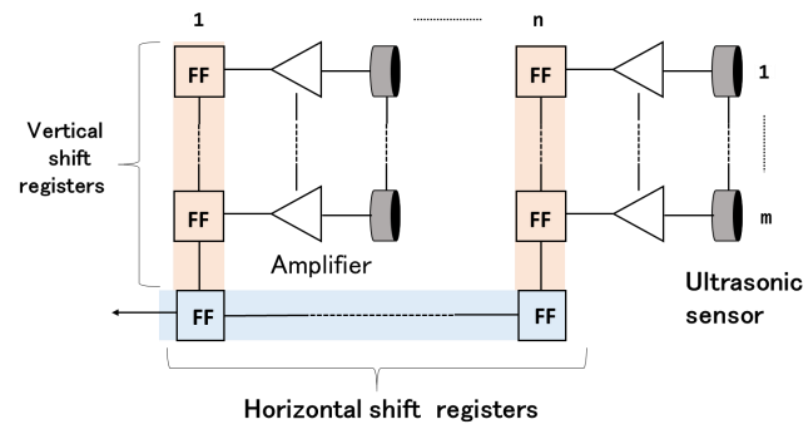

Fig. 1. Structure of Proposed 2-D Ultrasonic Sensor Array 
circuits would cause unexpected errors. All signals from each ultrasonic sensor must be amplified. Considering that ultrasonic sensors attached analog circuits are populated, analog circuits must be small-scale and simple. We attempt to realize analog circuits only by amplifier circuits to simplify the analog-digital interface.

In this paper, we first present the design of the proposed analog to digital interface. Then we describe a prototype system with the interface to verify the practical execution of measuring distance. After explaining setup of the measurement, we finally provide the result and discuss them.

\section{Analog to Digital Interface}

Fig. 2 shows the organization of the analog to digital interface for single ultrasonic sensor and corresponds to the parts in which outputs from each ultrasonic sensor are input into flip-flops in Fig. 1. As interface to the analog circuits, SR-FFs in the digital circuits capture amplified signals in the analog circuits. Fig. 3 shows the conceptual waveform representing the relation of input and output in the SR-FF. When input voltage reaches $V_{I H}$ of SR-FFs, the SR-FF is set. So flip-flops can capture signals from amplifiers directly. This saves other analog circuits such as comparators and smoothing circuits. Regarding the amplifier circuits, we constructed non- inverting amplifier circuits with op-amps. We added $C_{2}, R_{4}, R_{5}$ to standard non-inverting amplifier circuits. Bias voltage is set by $R_{4}, R_{5}$ and direct current is cut by $C_{2}$. The amplified voltage with bias is converted to nonbias voltage by $C_{3} . R_{6}$ is set for impedance matching. We adjusted parameters of the each element like Table. 1.

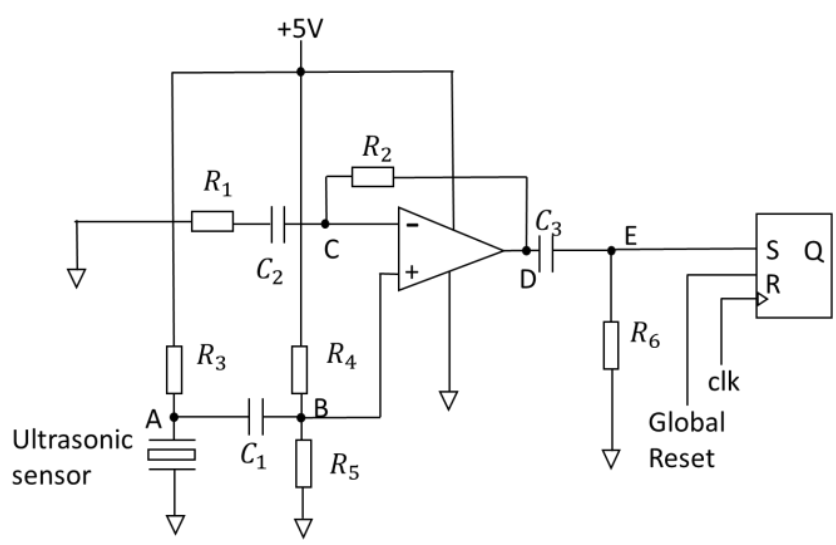

Fig. 2. Analog to Digital Interface
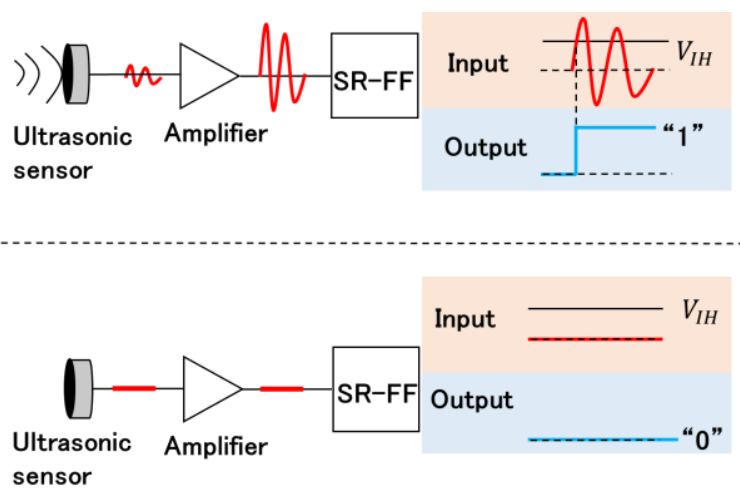

Fig. 3. Input and output in the SR-FF

Table 1. Parameter summary

\begin{tabular}{|c|c|}
\hline Parameter & Value \\
\hline$R_{1}(\mathrm{~K} \Omega)$ & 10 \\
\hline$R_{2}(\mathrm{M} \Omega)$ & 2.2 \\
\hline$R_{3}(\mathrm{~K} \Omega)$ & 22 \\
\hline$R_{4}(\mathrm{~K} \Omega)$ & 100 \\
\hline$R_{5}(\mathrm{~K} \Omega)$ & 100 \\
\hline$R_{6}(\mathrm{M} \Omega)$ & 1 \\
\hline$C_{1}(\mathrm{nF})$ & 100 \\
\hline$C_{2}(\mathrm{nF})$ & 470 \\
\hline$C_{3}(\mathrm{nF})$ & 100 \\
\hline
\end{tabular}

\section{Set up of Demonstration}

\subsection{Prototype}

We measured distance to verify the operation of the developed analog to digital interface. As shown in Fig. 4, the prototype consists of ultrasonic speakers (UT1612MPR, UT1007-Z325R), ultrasonic sensors (UR1612MPR), analog circuits, digital circuits and an oscilloscope. The ultrasonic speaker is driven by pulses generated in a FPGA (Zynq7000). The driven frequency is $40 \mathrm{KHz}$. The ultrasonic sensors are connected to the analog circuits. The signals are processed in the analog circuits as noted above. We designed SR-FFs that is interface to the analog circuits in the FPGA. Outputs from each SR-FF are connected to an oscilloscope to confirm times to be set in each SR-FF. 


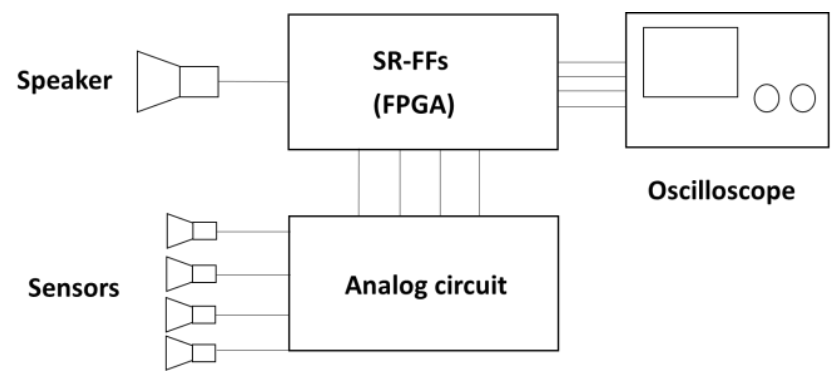

Fig. 4. Prototype

\section{2 placement}

As a measured objects, we used wooden blocks. The size is $30 \mathrm{~mm} \times 30 \mathrm{~mm} \times 30 \mathrm{~mm}$. Considering the directivity of the sensors and the speakers, the four objects were set. One speaker transmits waves and the reflected waves at the each object receive at the sensors placed on the opposite side of the objects. First, we placed the objects and speakers in one direction $\mathrm{x}(1 \times 4)$ like Fig. 5.Then, we placed these objects in two directions $x, y(2 \times 2)$ like Fig. 6 . Each object is placed in 2 patters (the distance from sensors is $11 \mathrm{~mm}$ or $26 \mathrm{~mm}$ ). So we placed four objects in 16 patterns and verified the relations of each position.

\subsection{Measuring Method}

The time difference of set times in each SR-FF connected to sensors represents the time difference of arrival waves. Each sensor receive same waves transmitted from a speaker. So relative distance of each object can be calculate by comparing set times in each SR-FFs. Fig. 7(a) shows the outputs of four SR-FFs when four objects are placed in different positions like Fig. 7(b). We decided a time reference

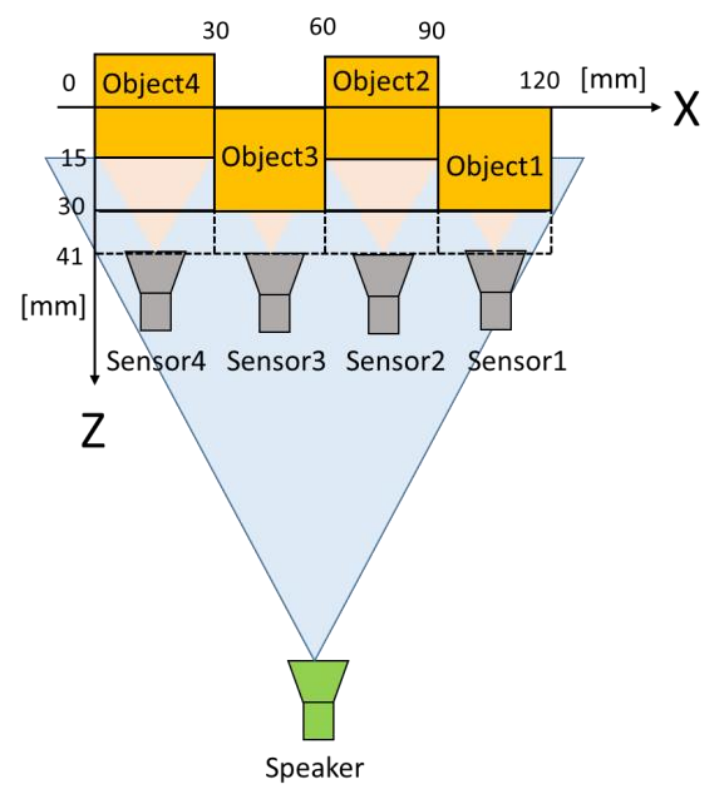

Fig. 5. The placement in $1 \times 4$

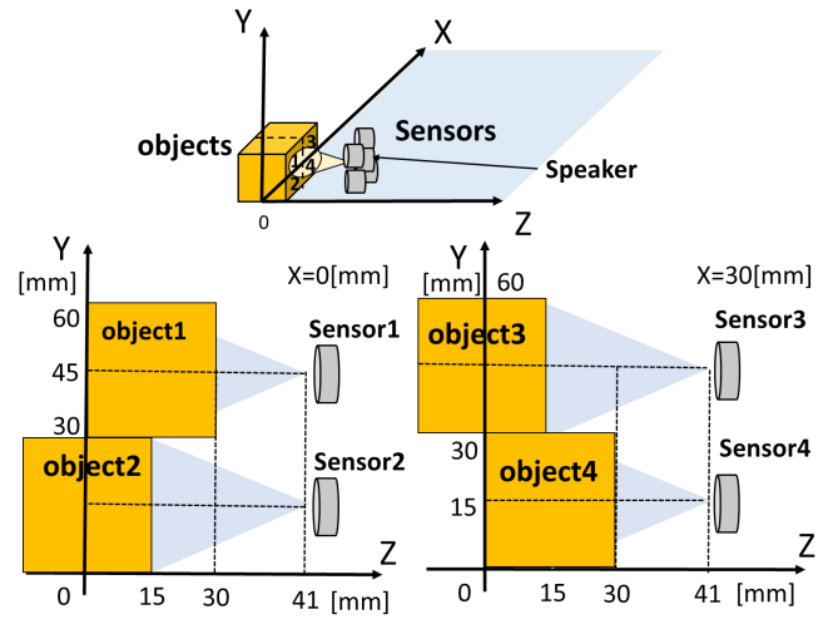

Fig. 6. The placement in $2 \times 2$

(0) to be set in the flip-flop connected to senser1. Time difference $(\alpha, \beta, \gamma)$ between set times in each SR-FF is measured. The distance from object1 to each object (a, b, c) can be calculated by the time difference. If the sound speed is $340 \mathrm{~m} / \mathrm{s}$, the relative distance can be calculated by following expressions.

$$
\begin{aligned}
& a[m]=(340 \times \alpha[s]) / 2 \\
& b[m]=(340 \times \beta[s]) / 2 \\
& c[m]=(340 \times \gamma[s]) / 2
\end{aligned}
$$

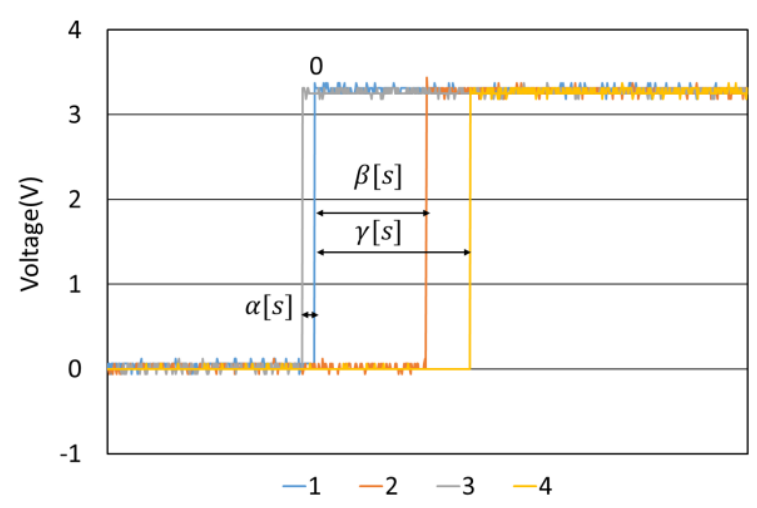

(a) Outputs from the SR-FFs

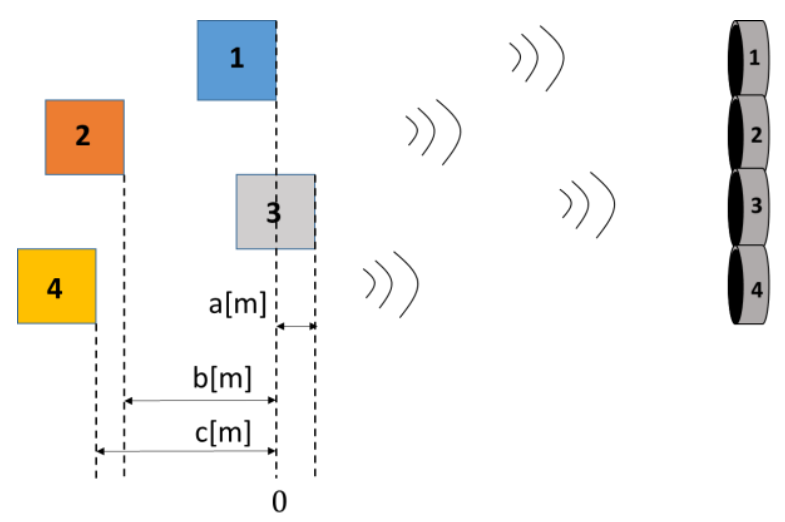

(b) Placement of objects

Fig. 7. The relation of distances and times 


\section{Result of Demonstration}

Fig. 8 and Fig. 9 show the experimental results. The placements illustrated in Fig. 5 and Fig. 6 correspond to the result shown in Fig. 8 and Fig. 9. The relative distance as a reference point of object 1 is $0 \mathrm{~mm}$ or $15 \mathrm{~mm}$. Fig. 8(a) and Fig. 9(a) show the amplified waves that were received in each sensor. First, the waves reflected at the objects placed in nearer to sensors were received and then the reflected waves from further objects were received. When the waves reached at $V_{I H}$ of the flip-flops $(2.0 \mathrm{~V})$, the SR-FFs were set like Fig. 8(b) and Fig. 9(b). A time reference to be set in the flip-flop connected to senser 1 was decided and the relative times were measured by set times of each SR-FF. The relative distance of each object was calculated by the set times. Table 2 and table 3 show the relation of times and distance. We tried to reconstruct the objects using the distance to confirm the form of the objects like Fig. 8(c) and Fig. 9(c). In these placement of the objects, the relative

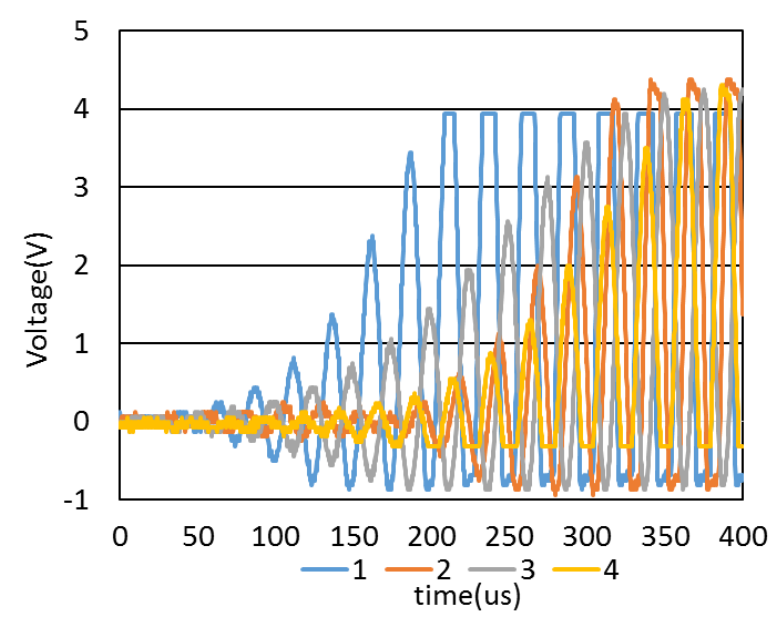

(a) Amplified signal
Table 2. The relation of times and distance in $1 \times 4$

\begin{tabular}{|l|l|l|}
\hline & $\Delta \mathrm{t}[\mathrm{us}]$ & $\Delta \mathrm{d}[\mathrm{mm}]$ \\
\hline 1 & & \\
\hline 2 & -149.36 & -25.3912 \\
\hline 3 & -30.92 & -5.2564 \\
\hline 4 & -151.48 & -25.7516 \\
\hline
\end{tabular}

Table 3 . The relation of times and distance in $2 \times 2$

\begin{tabular}{|l|l|l|}
\hline & $\Delta \mathrm{t}[\mathrm{us}]$ & $\Delta \mathrm{d}[\mathrm{mm}]$ \\
\hline 1 & & \\
\hline 2 & -97.25 & -16.5325 \\
\hline 3 & -77.6 & -13.192 \\
\hline 4 & -0.15 & -0.0255 \\
\hline
\end{tabular}

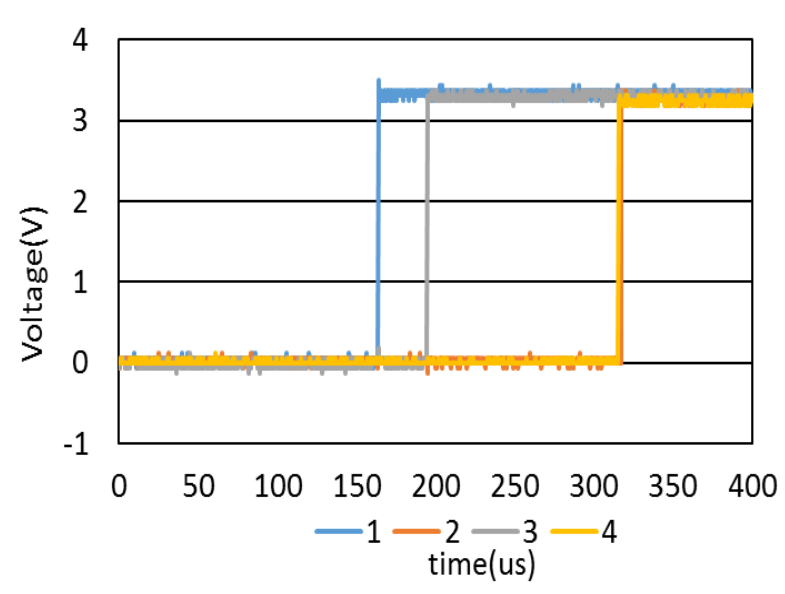

(b) Outputs from the SR-FFs

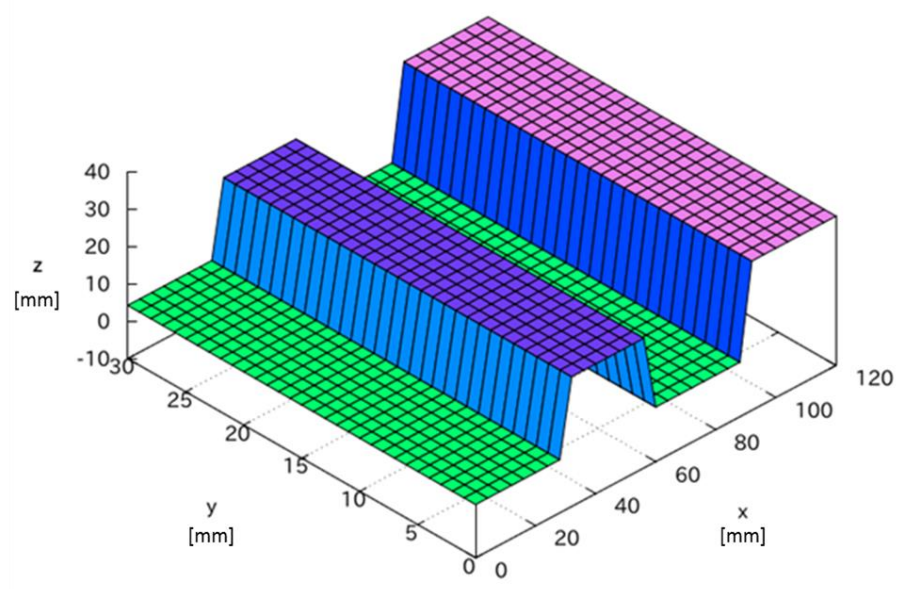

(c) Reconstructed image

Fig. 8. Experimental Result in $1 \times 4$ 


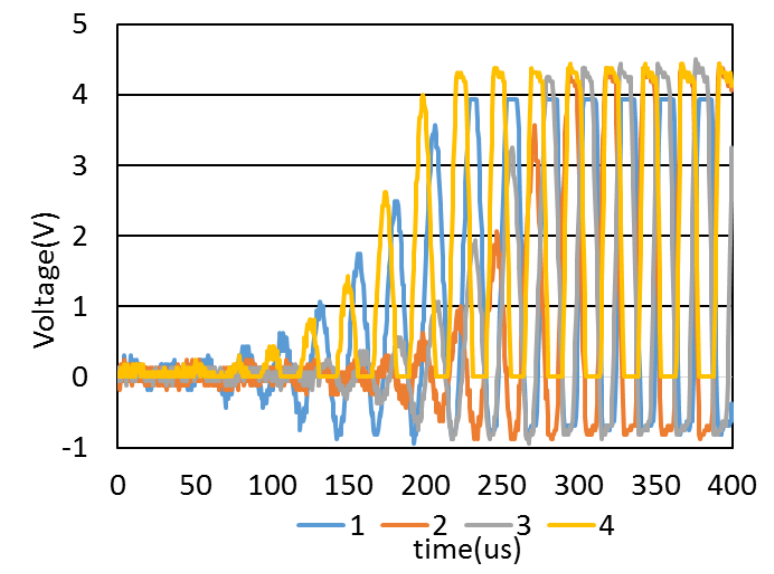

(a) Amplified signal

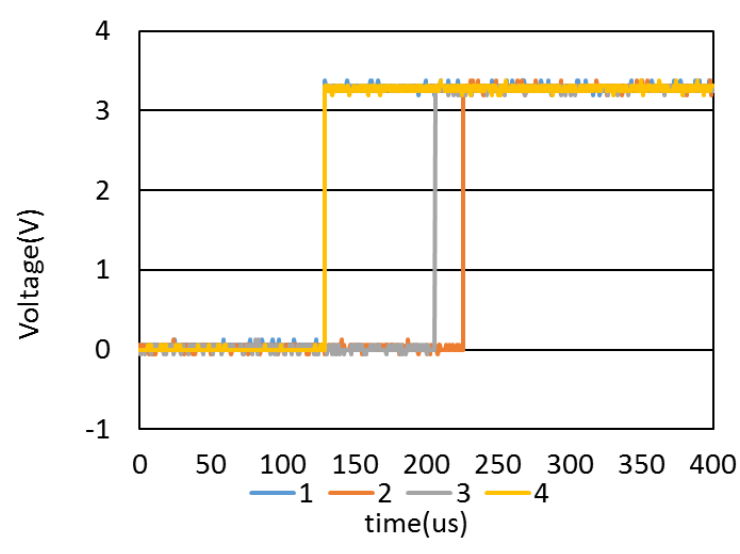

(b) Outputs from the SR-FFs

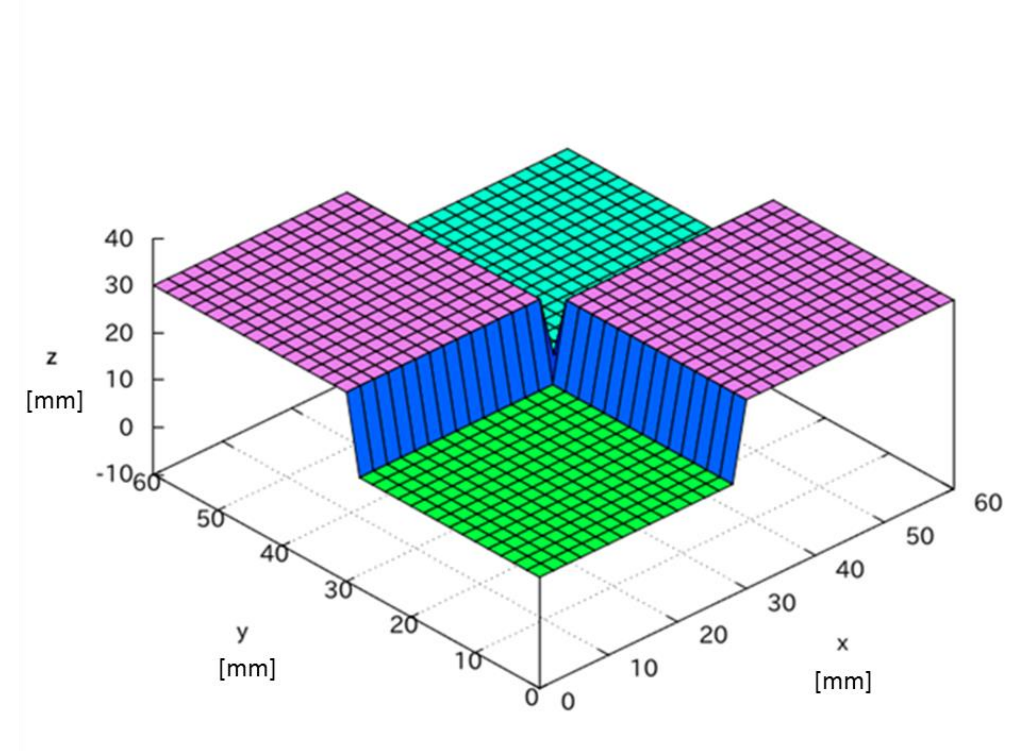

(c) Reconstructed image

Fig. 9. Experimental Result in $2 \times 2$

distance must be $0 \mathrm{~mm}$ or $15 \mathrm{~mm}$. However, the errors occurred in these measurement. One of the reason is that the frequency of the used ultrasonic wave is low. In these measurement, the operation frequency of the speaker was 40 $\mathrm{KHz}$. In measuring distance with ultrasonic wave, the distance that is shorter than wavelength of the used ultrasonic sound cannot be measured. The $40 \mathrm{KHz}$ wavelength in the air is $8.5 \mathrm{~mm}$. So the distance resolution is $8.5 \mathrm{~mm}$ and the distance can be measured only every $8.5 \mathrm{mms}$. This might cause errors in these measuring demonstrations.

After analyzing other 16 patterns in each placement, the distance could be measured without contradicting the relation of the position. Thus, we got a possibility to reconstruct the surface of the object for 3-D imaging system.

\section{Conclusions}

We have developed an analog to digital interface for scalable ultrasonic sensor array. The elements are only amplifiers and flip-flops. Amplified signals from ultrasonic sensor are input into flip-flops directly. After that we performed the measuring experiments using a prototype with the developed interface. The relative distance was be calculated by the set times of each flip-flop.

Considering that this structure is applied to real applications, the accuracy of the measured distance must be required. The higher frequency of ultrasonic sound is, the higher directivity and resolution are. As future work, we will improve the analog to digital interface to operate in high frequency. Then we will drive the speaker and sensors continuously to track the moving objects. Finally, we will try to realize 3-D imaging system using our proposal. 


\section{References}

(1) Yasutsugu Seo, Keisuke Hashimoto, Ryoichi Kanda, Satoru, Tezuka, Nobuyuki Iwata, Shinichi Hashimoto, Yoichi Ogasawara, Yasuhiko Abe, Hironobu Hongo, and Yasuo Miyajima : "Development of real-time threedimensional ultrasound imaging system", Acoustical Society of Japan, Vol. 61, No. 3, pp. 109-117, 2005 in Japanese

(2) Motohisa Abe, Hirokazu Karasawa : "MatrixeyeTM Portable 3D Ultrasonic Inspection System", TOSHIBA REVIEW, Vol. 60, No. 4, pp. 48-51, 2005

(3) Ira O. Wygant, Xuefeng Zhuang, David T. Yeh, Omer Oralkan, A. Sanli Ergun, Mustafa Karaman, Butrus T. Khuri-yakub : "Integration of 2D CMUT arrays with front-end electronics for volumetric ultrasound imaging", IEEE Transactions on Ultrasonic, Ferroelectrics, and Frequency Control, Vol. 55, No. 2, pp. 327-342, 2008

(4) Nakahata Kazuyuki, Horiguchi Takashi, Takamoto Tatsunao : "3D Reconstruction of Internal Flaws by FSAP Technique with Ultrasonic Matrix Array Transducer", IEICE Technical Report, Vol. 114, No. 464, pp. 23-27, 2015 in Japanese

(5) Anshuman Bhuyan, Jung Woo Choe, Byung Chul Lee, Ira O. Wygant, Amin Nikoozadeh, Ömer Oralkan, Butrus T. Khuri-Yakub : "Integrated Circuits for Volumetric Ultrasound Imaging With 2-D CMUT Arrays", IEEE Transactions on Biomedical Circuits and Systems, Vol. 7, No. 6, pp. 796-804, 2013

(6) Yuichi Morita, Yasunobui Takaichi, and Akira Yamawaki :"A Scalable Organization of 2-D Ultrasonic Sensor Array", Proceedings of the 4th IIAE International Conference on Intelligent Systems and Image Processing 2016, pp. 155-159, 2016 